Series A

I. MATHEMATICA

437

\title{
ZUR VERALLGEMEINERUNG DES POISSONSCHEN SATZES
}

VON

KARL WILHELM BAUER

HELSINKI 1968

S U O M A A I N E T TEDEAKATEMIA

doi:10.5186/aasfm.1969.437 
Am 15. September 1967 vorgelegt von P. J. Myrberg und R. Nevanlinna 


\section{Zur Verallgemeinerung des Poissonschen Satzes}

1. Einführung. In der vorliegenden Arbeit wird der Poissonsche Satz für den Fall der Differentialgleichung

$$
(1+\varepsilon z \bar{z})^{2} w_{z \bar{z}}+\varepsilon n(n+1) w=0, \quad \varepsilon= \pm 1, \quad n \in N,
$$

behandelt ${ }^{1}$ ). Diese Differentialgleichung, die sich mit $n$ bzw. $\varepsilon=0$ auf die Potentialgleichung für die Ebene reduziert, steht in enger Beziehung zur Wellen- bzw. Potentialgleichung für drei Raumdimensionen (vgl. [2] [3]). Sie ist invariant gegenüber Kugeldrehungen im Falle $\varepsilon=+1$ bzw. Automorphismen des Einheitskreises im Falle $\varepsilon=-1$, da mit

$$
(1+\varepsilon z \bar{z})^{2} \frac{\partial^{2}}{\partial z \partial \bar{z}}
$$

der bezüglich der betreffenden Gruppe von Isometrien invariante BeltramiOperator vorliegt. In [8] wurden die Lösungen der Differentialgleichung

$$
(1+\varepsilon z \bar{z})^{2} w_{z \bar{z}}+\lambda w=0
$$

für den Fall $\varepsilon=-1$ (umgeschrieben auf die obere Halbebene) im Hinblick auf Grenzkreisgruppen erster Art untersucht. In [2] wurde unter anderem eine Darstellung für die in einfach zusammenhängenden Gebieten der Zahlenkugel $(\varepsilon=+1)$ bzw. des Einheitskreises $(\varepsilon=-1)$ definierten komplexwertigen bzw. reellwertigen Lösungen von (1) ermittelt. Das Verhalten der Lösungen von (1) in der Nähe isolierter Singularitäten wurde in [4] untersucht, in [1] wurde eine der Differentialgleichung (1) zugeordnete Funktionentheorie entwickelt.

Während das Dirichlet-Problem für den Kreis $z \bar{z}<R^{2}$ im Falle $\varepsilon=-1$ eindeutig lösbar ist, trifft dies bekanntlich im Falle $\varepsilon=+1$ im allgemeinen nicht $z u$. Es zeigt sich jedoch, dass sich auch für $\varepsilon=+1$ genauere Ergebnisse gewinnen lassen. Es erscheint besonders bemerkenswert, dass unsere Methode in beiden Fällen, also auch im Falle $\varepsilon=+1$, zu einer Verallgemeinerung der Poissonschen Formel und damit zur Lösung des DirichletProblems für den Kreis $z \bar{z}<R^{2}$ führt, wobei im Falle $\varepsilon=+1$ gewisse

1) Mit $\boldsymbol{N}, \boldsymbol{R}$ bzw. $\boldsymbol{C}$ wird die Menge der natürlichen, reellen bzw. komplexen Zahlen bezeichnet. 
Kreisradien ausgenommen werden, deren Anzahl und Grösse vom Parameter $n$ abhängig ist ${ }^{2}$ ). Hierbei ziehen wir einen allgemeinen Darstellungssatz [2] für die komplexwertigen bzw. reellwertigen Lösungen von (1) heran. Die Ausnahmeradien ergeben sich als Nullstellen gewisser Polynome, die explizit angegeben werden; ferner lassen sich in diesen Ausnahmefällen die möglichen Differenzfunktionen zweier Lösungen des Dirichlet-Problems explizit angeben. Es wird sodann eine Singularitätenfunktion und eine Grundlösung definiert und ein Verfahren zur Bestimmung der Greenschen Funktion angegeben. Liegt die Singularität in $\zeta=0$, so hat die Greensche Funktion eine besonders einfache Gestalt; hier gilt (vgl. 5):

$$
g(0,0 ; z, \bar{z})=-\frac{1}{4 \pi}\{(E f)(z)+c j .\}
$$

mit

$$
f(z)=z^{n} \log \frac{z}{R}+C z^{n}, \quad C \in R
$$

Im allgemeinen Fall $(\zeta \neq 0)$ erhält man die Greensche Funktion unter Verwendung einer in $|z| \leqq R$ definierten Lösung einer inhomogenen. Eulerschen Differentialgleichung der Ordnung $n$. Es wird sodann für beide Werte von $\varepsilon$, mit Ausnahme der oben genannten Radien im Falle $\varepsilon=+1$, eine verallgemeinerte Poissonsche Formel hergeleitet und ein verallgemeinerter Poissonscher Satz bewiesen. Unter Verwendung cines Satzes über die komplexwertigen Lösungen von (1) mit gleichem Realteil wird abschliessend die aus der klassischen Funktionentheorie bekannte Schwarzsche Formel für die Darstellung einer in $z<R$ holomorphen Funktion mit Hilfe der Randwerte des Realteils für eine entspreschende Klasse von Lösungen der Differentialgleichung (1) verallgemeinert. Dabei zeigt sich, dass eine solche Funktion bei Vorgabe des Imaginärteils in $2 n+1$ geeignet gewählten Punkten eindeutig bestimmt ist, was für $n=0$ genau dem klassischen Fall entspricht.

2. Grundlegende Darstellungssätze. Im Himblick auf die folgenden Untersuchungen werden zunächst einige der in den Arbeiten [1] bis [4] gewonnenen Ergebnisse zusammengestellt. Dabei verwenden wir die folgenden Differentialoperatoren

$\Rightarrow$ In [5] wird unter Verwendung von Integraldarstellungen [9] das Dirichlet. Problem für den Einheitskreis im Falle der Differentialgleichung (1) mit $\varepsilon=+1$ behandelt. Das hier diskutierte Problem $(R=1)$ stellt einen der Fällo dar, in denen das Randwertproblem nicht eindeutig lösbar ist. 
(2)

$$
D_{\varepsilon}=(1+\varepsilon z \bar{z})^{2} \frac{\partial}{\partial \bar{z}}, \quad D_{\varepsilon}^{2}=(1+\varepsilon z \bar{z})^{2}\left((1+\varepsilon z \bar{z})^{2} \frac{\partial}{\partial \bar{z}}\right), \ldots
$$

$$
D_{\varepsilon}^{0} w=w
$$

$$
\underset{z}{E}=\sum_{k=0}^{n} A_{k} \bar{\tau}^{n-k} \frac{\partial^{k}}{\partial z^{k}}
$$

mit

$$
A_{k}=\frac{(-\varepsilon)^{n-k}(2 n-k) !}{k !(n-k) !}, \quad \tau=\frac{z}{1+\varepsilon z \bar{z}}
$$

und

$$
\underset{z}{E}(g(z))=(E g)(z), \quad g(z) \text { holomorph. }
$$

Satz I. a) $Z u$ jeder in einem einfach zusammenhängenden (endlichen) Gebiet $G$ der Riemannschen Zahlenkugel $(\varepsilon=+1)$ bzw. des Einheitskreises ( $\varepsilon=-1$ ) definierten Lösung von (1) gibt es in $G$ holomorphe Funktionen $h(z)$ und $f(z)$, so dass

$$
w=(E h)(z)+\overline{(E f)(z)} .
$$

b) Umgekehrt stellt (4) für jedes Paar von in $G$ holomorphen Funktionen $h(z)$ und $f(z)$ eine Lösung von (1) in G dar. (Vgl. [2].)

Satz II. a) Zu jeder in einem einfach zusammenhängenden (endlichen) Gebiet $G$ der Riemannschen Zahlenkugel bzw. des Einheitskreises definierten reellen Lösung von (1) gibt es in $G$ holomorphe Funktionen $h(z)$, so dass ${ }^{3}$ )

$$
w=(E h)(z)+\mathrm{cj} .
$$

b) Umgekehrt stellt (5) für jede in G holomorphe Funktion $h(z)$ eine reellwertige Lösung von (1) dar. (Vgl. [2].)

Satz III. a) Zwei in einem einfach zusammenhängenden (endlichen) Gebiet $G$ der Riemannschen Zahlenkugel bzw. des Einheitskreises definierte Lösungen

$$
w_{1}=\left(E h_{1}\right)(z), \quad w_{2}=\left(E h_{2}\right)(z)
$$

von (1) stimmen genau dann in ihren Realteilen überein, wenn die Erzeugenden $h_{1}(z)$ und $h_{2}(z)$ der Bedingung

$$
h_{2}(z)=h_{1}(z)+P(z)
$$

3) Die hier verwendete Schreibweise besagt, dass der zum voraufgehenden konjugiert komplexe Ausdruck addiert werden soll. 
genügen. Dabei stellt $P(z)$ ein Polynom vom Grade $2 n$ in z mit

$$
P(z)+(-\varepsilon)^{n} z^{2 n} \overline{P\left(\frac{-\varepsilon}{\bar{z}}\right)}=0
$$

dar. (Vgl. [1].)

b) $Z u$ jeder in $G$ definierten Lösung

$$
w=(E h)(z)
$$

ist die Erzeugende durch

$$
h(z)=\frac{(-\varepsilon)^{n}}{(2 n) !} D_{*}^{n} w
$$

eindeutig bestimmt. (Vgl. [3].)

Satz IV. a) Jede in $z \bar{z} \leqq R^{2}<1$ definierte und auf $z \bar{z}=R^{2}$ konstante Lösung der Differentialgleichung

$$
(1-z \bar{z})^{2} w_{z \bar{z}}-n(n+1) w=0, \quad n \in N,
$$

reduziert sich auf eine Funktion

$$
w=(E h)(z) \quad \text { mit } \quad h(z)=a z^{n} .
$$

b) Eine derart bestimmte Lösung ist damit im gesamten Einheitshreis definiert.

c) Falls $w=C \in C$ auf $z \bar{z}=R^{2}$, so folgt

$$
a=\frac{C}{n ! \sum_{k=0}^{n}\left(\begin{array}{c}
2 n-k \\
n
\end{array}\right)\left(\begin{array}{l}
n \\
k
\end{array}\right)\left(\frac{R^{2}}{1-R^{2}}\right)^{n-k} \cdot}
$$

(Vgl. [1].)

Satz V. a) Jede in $z \bar{z} \leqq R^{2}$ definierte und auf $z \bar{z}=R^{2}$ konstante Lösung der Differentialgleichung

$$
(1+z \bar{z})^{2} w_{z \bar{z}}+n(n+1) w=0 . \quad n \in N .
$$

reduziert sich auf eine Funktion

$$
w=(E h)(z) \quad \text { mit } \quad h(z)=\sum_{\mu=1}^{2 n-1} a_{\mu} z^{\prime \prime} .
$$

b) Falls

$$
R_{n}(R)=\sum_{k=0}^{\mu} \frac{A_{k} R^{n+\mu-2 k}}{(\mu-k) !\left(1+R^{2}\right)^{n-k}}=0
$$


für ein festes $\mu \in\{1,2, \ldots, n\}$, so können die Koeffizienten $a_{\mu}$ und $a_{2 n-\mu}$ beliebig gewählt werden.

Falls $R_{\mu}(R) \neq 0$ für $\mu \in\{1,2, \ldots, n-1\}$, so sind die Koeffizienten $a_{\mu}$ und $a_{2 n-\mu}$ gleich Null zu setzen.

Falls $R_{n}(R) \neq 0$ und $w=C \in C$ auf $z \bar{z}=R^{2}$, so gilt

$$
a_{n}=\frac{C}{n ! R_{n}(R)} .
$$

c) Im Falle $R_{n}(R)=0$ ist lediglich $w=0$ auf $z \bar{z}=R^{2}$ möglich.

d) Im Falle einer reellwertigen Lösung unterliegen die Koeffizienten $a_{n}, \mu=1, \ldots, 2 n-1$, der zusätzlichen Bedingung

(Vgl. [1].)

$$
a_{\mu}=(-1)^{n+\mu} \overline{a_{2 n-\mu}} .
$$

Satz VI. Jede im Kreisring

$$
T=\left\{z\left|\quad 0 \leqq \varrho_{1}<\right| z-z_{0} \mid<\varrho_{2}\right\}
$$

definierte und eindeutige reellwertige Lösung der Differentialgleichung (1) lässt sich in $T$ gemäss

$$
w=(E h)(z)+\mathrm{cj} .
$$

mit der Erzeugenden

$$
h(z)=h_{1}(z)+S_{1}(z) \log \left(z-z_{0}\right)
$$

darstellen, wobei $h_{1}(z)$ in $T$ holomorph und eindeutig ist, während $S_{1}(z)$ ein Polynom vom Grade $2 n$ in $z$ bezeichnet, welches der Bedingung

$$
S_{1}(z)=(-\varepsilon)^{n} z^{2 n} S_{1}\left(\frac{-\varepsilon}{\bar{z}}\right)
$$

genügt. (Vgl. [4].)

3. Diskussion der eindeutigen Lösbarkeit des Dirichlet-Problems für den Kreis. Wir diskutieren zunächst die Frage, ob das DirichletProblem für den Kreis

$$
K=\left\{z \mid z \bar{z}<R^{2}\right\}
$$

im Falle der Differentialgleichung (1) eindeutig lösbar ist. Liegen zwei (reellwertige) Lösungen $w_{1}$ und $w_{2}$ für dieses Problem vor, so stellt

$$
w=w_{2}-w_{1}
$$


eine Lösung von (1) in $K$ dar, die auf dem Rande $\dot{K}$ verschwindet. Jede derartige Lösung lässt sich jedoch im Falle $\varepsilon=-1$ nach Satz IV durch

$$
w=(E h)(z) \quad \text { mit } \quad h(z)=a z^{n}
$$

darstellen. Wegen $w \mid \dot{K} \equiv 0$ folgt jedoch unter Verwendung von (7) $a=0$ und damit

$$
w \equiv 0 \quad \text { bzw. } \quad w_{1} \equiv w_{2} .
$$

Das heisst, das gestellte Dirichlet-Problem hat für $\varepsilon=-1$ höchstens eine Lösung.

Im Falle $\varepsilon=+1$ lässt sich $w=w_{2}-w_{1}$ nach Satz $V$ gemäss (8) darstellen. Falls für einen gegebenen Radius $R$ die Grösse $R_{\mu}(R)$ (vgl. (9)) für ein festes $\mu \in\{1,2, \ldots, n\}$ verschwindet, so kann der betreffende Koeffizient $a_{\mu}$ beliebig gewählt werden, während

$$
a_{2 n-\mu}=(-1)^{2 n-\mu} \overline{a_{\mu}}
$$

zu setzen ist; das heisst, es gibt unendlich viele Lösungen

$$
w=w_{2}-w_{1} \quad \text { mit } \quad w \mid \dot{K} \equiv 0,
$$

und das gestellte Dirichlet-Problem ist im Falle $\varepsilon=+1$ generell nicht eindeutig lösbar. Wählt man jedoch einen Kreis $K$, so dass für den verwendeten Radius $R$ alle

$$
R_{\mu}(R) \neq 0 \quad \text { für } \quad 1 \leqq \mu \leqq n,
$$

so sind in (8) alle Koeffizienten $a_{\mu}$ gleich Null zu setzen. Damit gilt wiederum

$$
w \equiv 0 \quad \text { bzw. } \quad w_{1} \equiv w_{2} .
$$

Wir fassen die Ergebnisse im folgenden Satz zusammen.

Satz 1. Es sei

$$
K=\left\{z \mid z \bar{z}<R^{2}\right\}
$$

und im Falle $\varepsilon=+1$

$$
R_{\mu}(R) \neq 0 \quad \text { für } \quad 1 \leqq \mu \leqq n .
$$

Dann hat das Dirichlet-Problem für die Differentialgleichung (1) und den Kreis $K$ höchstens eine Lösung.

4. Singularitätenfunktion und Grundlösung ${ }^{4}$ ). Wir definieren nun die für den vorliegenden Fall zu verwendende Singularitätenfunktion und die Grundlösung. Dabei werden hier und im folgenden Formulierungen

$\left.{ }^{4}\right)$ Wir verwenden hier die bei Hellwig [6] übliche Bezeichnungsweise. 
gewählt, die sich mit $\varepsilon=n=0$ stets auf die klassischen Ergebnisse der Potentialtheorie reduzieren.

Nach Satz VI lässt sich jede in einem Kreisring eindeutige reellwertige Lösung von (1) gemäss (10) darstellen, wobei die Erzeugende den Bedingungen (11) und (12) genügt. Darüber hinaus wurde in [4] gezeigt, dass: sich jede in $0<\left|z-z_{0}\right|<\varrho$ definierte reellwertige Lösung von (1), die in $z=z_{0}$ eine isolierte Singularität mit logarithmischem Hauptglied der asymptotischen Entwicklung besitzt, gemäss (10) mit

$$
h(z)=S_{2}(z) \log \left(z-z_{0}\right)
$$

und

$$
\begin{aligned}
S_{2}(z) & =C\left(\frac{\left(z-z_{0}\right)\left(1+\varepsilon \bar{z}_{0} z\right)}{1+\varepsilon z_{0} \bar{z}_{0}}\right)^{n} \\
& =C \sum_{\mu=0}^{n}\left(\begin{array}{l}
n \\
\mu
\end{array}\right)\left(\frac{\varepsilon \bar{z}_{0}}{1+\varepsilon z_{0} \bar{z}_{0}}\right)^{\mu}\left(z-z_{0}\right)^{n+\mu}, \quad C \in R,
\end{aligned}
$$

darstellen lässt. Mit Rücksicht auf dieses Ergebnis definieren wir die Singularitätenfunktion wie folgt:

Definition 1. Die für $z \neq \zeta$ definierte Lösung

$$
s(\zeta, \bar{\zeta} ; z, \bar{z})=-\frac{1}{4 \pi}\{(E h)(z)+\mathrm{cj} \cdot\}
$$

mit

$$
h(z)=\frac{(z-\zeta)^{n}(1+\varepsilon \bar{\zeta} z)^{n}}{(1+\varepsilon \zeta \bar{\zeta})^{n}} \log (z-\zeta)=S(\zeta, \bar{\zeta} ; z) \log (z-\zeta)
$$

heisst Singularitätenfunktion zu (1).

Man erhält explizit:

$$
s=-\frac{1}{2 \pi}(E S)(z) \log |z-\zeta|+T_{1}+\overline{T_{1}}
$$

mit

$$
T_{1}=-\frac{1}{4 \pi} \sum_{k=1 \rrbracket}^{n} A_{k} \bar{\tau}^{n-k} \sum_{\mu=1}^{k}\left(\begin{array}{l}
k \\
\mu
\end{array}\right) \frac{\partial^{k-\mu} S}{\partial z^{k-\mu}} \frac{(\mu-1) !(-1)^{\mu-1}}{(z-\zeta)^{\mu}} .
$$

Dabei stellt $(E S)(z)$ eine reellwertige Funktion dar (vgl. [4]); der Summand $T_{1}$ ist wegen (14) an der Stelle $z=\zeta$ differenzierbar.

Im Falle $\varepsilon=n=0$ reduziert sich der Operator $E$ angewandt auf $h(z)$ auf die Funktion $h(z)$; damit gilt hier: 


$$
\begin{aligned}
(E h)(z) & =h(z)=-\frac{1}{4 \pi}\{\log (z-\zeta)+\mathrm{cj} \cdot\} \\
& =-\frac{1}{2 \pi} \log |z-\zeta| .
\end{aligned}
$$

Bezeichnet $G$ ein Normalgebiet, so sind auf $\dot{G}$ bzw. $G$ der Gausssche Integralsatz und die Greenschen Formeln anwendbar. Es sei $\zeta \in G$ und $\Phi=\Phi(\zeta, \bar{\zeta} ; z, \bar{z})$ eine reellwertige Funktion mit folgenden Eigenschaften:

1. $\bar{\Phi} \in C^{1}$ in $\bar{G}$.

2. $\Phi \in C^{2}$ in $G$ und

3. $(1+\varepsilon z \bar{z})^{2} \Phi_{z \bar{z}}+\varepsilon n(n+1) \Phi=0$ in $G$.

Definition 2. Die für $\approx=\zeta$ definierte Funktion

$$
\gamma(\zeta, \bar{\zeta} ; z, \bar{z})=s(\zeta, \bar{\zeta} ; z, \bar{z})+\Phi(\zeta, \bar{\zeta} ; z, \bar{z})
$$

heisst Grundlösung von (1) bezüglich $G$.

Es sei $v(z, \bar{z})$ die äussere Normale von $\bar{G} ; u_{v}(z, \bar{z})$ bezeichne die Richtungsableitung der reeliwertigen Funktion $u(z, \bar{z})$ in Richtung der äusseren Normalen $v$. Dann erhält man für $u, v \in C^{1}$ in $\bar{G}$ und $u, v \in C^{2}$ in $G$ unter Verwendung der 2. Greenschen Formel

$$
\int_{G}(u \Delta v-v \Delta u) d x d y=\sum_{j=1}^{m} \int_{\dot{C}_{j}}\left(u v_{r_{j}}-v u_{r_{j}}\right) d s_{j}
$$

wobei $v_{j}$ die äussere Normale von $G$ auf $\dot{G}_{j}$ darstellt, $d_{s_{j}}$ das zn $\dot{G}_{j}$ gehörende Linienelement bezeichnet und jedes $G_{j}$ ein Normalgebiet ist. Unter Verwendung von (19) beweisen wir nun den folgenden

Satz 2. $G$ bezeichne cin Nomalgebiet mit

$$
G \subset K_{0}=\{z \mid z z<<1\}
$$

für $\varepsilon=-1$. Für die reellwertige Funktion $w(z, \bar{z})$ gelte:

1. $w \in C^{1}$ in $\bar{G}$,

2. $w \in C^{2}$ in $G$,

3. w sei Lösung von (1) in $G$,

4. $\zeta \in G$.

Dann gilt:

$$
w(\zeta, \bar{\zeta})=\int_{\dot{G}}\left(\gamma w_{\nu}-w \gamma_{\nu}\right) d s
$$


Beweis. Mit $\zeta$ enthält $G$ bei geeigneter Wahl von $\varrho$ auch den Kreis

$$
\bar{K}_{:}=\{z|| z-\zeta \mid \leqq \varrho\} .
$$

$\gamma$ wird für $z \rightarrow \zeta$ singulär; wir schneiden deshalb $\bar{K}_{\text {: }}$ aus $G$ aus und wenden auf $G-\bar{K}_{;}$die Formel (21) an. Dann gilt

$$
\begin{aligned}
& \int_{G-\bar{K}_{\dot{\zeta}}}(w \Delta \gamma-\gamma \Delta w) d x d y \\
& =\int_{\dot{\dot{G}}}\left(\gamma w_{\nu}-w \gamma_{\nu}\right) d s-\int_{|z-=|=v}\left(\gamma w_{v}-w \gamma_{v}\right) d s .
\end{aligned}
$$

Dabei bezeichnet $\nu$ im letzten Integral die äussere Normale von $\bar{K}_{\zeta}$. Da $\gamma$ und $w$ Lösungen von (1) in $G-\bar{K}_{;}$, gilt

$$
w \Delta \gamma-\gamma \Delta w=0
$$

und damit an Stelle von (23), wenn wir noch

berücksichtigen,

$$
\gamma=s+\Phi
$$

$$
\int_{\dot{G}}\left(\gamma w_{\nu}-w \gamma_{\nu}\right) d s=I_{1}+I_{2}
$$

mit

und

$$
I_{1}=\int_{|z-s|=0}\left(\Phi w_{v}-u \Phi_{v}\right) d s
$$

$$
I_{2}=\int_{\mid z-\zeta=0}\left(s w_{p}-w s_{v}\right) d s .
$$

In $I_{1}$ ist der Integrand für $|z-\zeta| \leqq \varrho$ stetig; damit folgt

$$
\lim _{\varrho \rightarrow 0} I_{1}=0 \text {. }
$$

Im Falle des Integrals $I_{2}$ gilt

$$
\int_{\mid z-\zeta==} s w_{\nu} d s=\varrho \int_{0}^{2 \pi} s(\zeta, \bar{\zeta} ; \zeta+\varrho v, \bar{\zeta}+\varrho \bar{v}) w_{\nu}(\zeta+\varrho v, \bar{\zeta}+\varrho \bar{v}) d \varphi
$$

und wegen (17) 


$$
\lim _{\varrho \rightarrow 0} \int_{|z-\zeta|=\varrho} s w_{\nu} d s=0 .
$$

Für die Richtungsableitung gilt

$$
s_{\nu}=\frac{1}{|z-\zeta|}\left\{(z-\zeta) s_{z}+(\bar{z}-\bar{\zeta}) s_{\bar{z}}\right\}
$$

und damit unter Verwendung von (17)

$$
s_{v}=\frac{z-\zeta}{\varrho}\left\{-\frac{1}{2 \pi}((E S)(z))_{z} \log |z-\zeta|-\frac{1}{4 \pi} \frac{(E S)(z)}{z-\zeta}+\left(T_{1}+\bar{T}_{1}\right)_{z}\right\}+\mathrm{cj} .
$$

Setzt man in

$(E S)(z)=\sum_{k=0}^{n} A_{k}\left(\frac{\bar{z}}{1+\varepsilon z \bar{z}}\right)^{n-k} \sum_{\mu=0}^{n}\left(\begin{array}{l}n \\ \mu\end{array}\right)\left(\frac{\varepsilon \bar{\zeta}}{1+\varepsilon \zeta \bar{\zeta}}\right)^{\mu} \frac{(n+\mu) !}{(n+\mu-k) !}(z-\zeta)^{n+\mu-k}$ $z=\zeta+\varrho v$, so verschwinden mit $\varrho \rightarrow 0$ alle Summanden mit Ausnahme des für $n+\mu-k=0$ entstehenden Terms. Damit gilt

$$
\lim _{\varrho \rightarrow 0}\left(s_{\nu} \varrho\right)=-\frac{1}{2 \pi},
$$

und es folgt

$$
\lim _{\varrho \rightarrow 0}\left\{-\int_{|z-\zeta|=\varrho} w s_{\nu} d s\right\}=w(\zeta, \bar{\zeta}) .
$$

Unter Verwendung von (25) bis (27) erhält man sodann die Aussage des Satzes 2.

5. Bestimmung der Greenschen Funktion. Wir konstruieren nun die Greensche Funktion $g$ für den Kreis

$$
\bar{K}=\left\{z \mid z \bar{z} \leqq R^{2}\right\}
$$

und setzen

$$
g(\zeta, \bar{\zeta} ; z, \bar{z})=-\frac{1}{4 \pi}\{(E f)(z)+\mathrm{cj} .\}
$$

mit

$$
f(z)=S(\zeta, \bar{\zeta} ; z) \log \frac{R(z-\zeta)}{\bar{\zeta} z-R^{2}}+\varphi(z) .
$$

Dabei ist die in $\bar{K}$ holomorphe Funktion $\varphi(z)$ so zu bestimmen, dass

$$
g \mid \dot{K} \equiv 0 \text {. }
$$


Die Greensche Funktion für den Kreis $K$ lautet also:

$$
g(\zeta, \bar{\zeta} ; z, \bar{z})=-\frac{1}{4 \pi}\{(E f)(z)+\mathrm{cj} .\}
$$

mit

$$
f(z)=S(\zeta, \bar{\zeta} ; z) \log \frac{R(z-\zeta)}{\zeta z-R^{2}}+\varphi(z)
$$

wobei

$$
\varphi(z)=\frac{1}{(1+\varepsilon \zeta \bar{\zeta})^{n}} \sum_{j=1}^{\infty} a_{j}(\zeta, \bar{\zeta}) z^{j}
$$

die oben bestimmte und in $|z| \leqq R$ gleichmässig konvergente Lösung von (41) darstellt. Dabei ist für den Radius $R$ des betrachteten Kreises $K$ im Falle $\varepsilon=+1$

$$
\sum_{k=0}^{j} A_{k}\left(\frac{R^{2}}{1+R^{2}}\right)^{n-k} \frac{1}{(j-k) !} \neq 0 \text { für } j=1, \ldots, n
$$

vorauszusetzen.

Im Falle $\zeta=0$ erhällt die Greensche Funktion eine besonders einfache Gestalt. Die Erzeugende lautet hier:

$$
f(z)=z^{n} \log \frac{z}{R}+\varphi(z) .
$$

Dann gilt

$$
-4 \pi g(0,0 ; z, \bar{z})=2\left(E z^{n}\right)(z) \log \frac{|z|}{R}+2 T_{4}+\{(E \varphi)(z)+\mathrm{cj} .\}
$$

mit

$$
T_{4}=n ! \sum_{k=1}^{n}\left(\begin{array}{c}
2 n-k \\
n
\end{array}\right)\left(\frac{-\varepsilon z \bar{z}}{1+\varepsilon z \bar{z}}\right)^{n-k} \sum_{\mu=1}^{k} \frac{(-1)^{\mu-1}}{\mu}\left(\begin{array}{c}
n \\
k-\mu
\end{array}\right) .
$$

Auf $|z|=R$ gilt also

$$
T_{4} \mid \dot{K}=C_{n}=\text { const. }
$$

Wir erhalten also, wenn wir

$$
\varphi(z)=C z^{n} \quad \text { mit } \quad C=-\frac{C_{n}}{D_{n}}
$$

und 


$$
D_{n}=n ! \sum_{k=1}^{n}\left(\begin{array}{c}
2 n-k \\
n
\end{array}\right)\left(\begin{array}{l}
n \\
k
\end{array}\right)\left(\frac{-\varepsilon R^{2}}{1+\varepsilon R^{2}}\right)^{n-k} \neq 0
$$

verwenden, die gesuchte Greensche Funktion in Falle $\zeta=0$.

6. Die Poissonsche Formel. Da es sich im vorliegenden Fall um ein selbstadjungiertes Randwertproblem handelt, ist die Greensche Funktion symmetrisch; es gilt also

$$
g(\zeta, \bar{\zeta} ; z, \bar{z})=g(z, \bar{z} ; \zeta, \bar{\zeta}) .
$$

Wir setzen in $(46)$

$$
f(z)=f(\zeta, z) \text { und } \varphi(z)=\varphi(\zeta, z),
$$

dann gilt

$$
-4 \pi g=\underset{z}{E}(f(\zeta, z))+\mathrm{cj.}=\underset{\zeta}{E(f(z, \zeta))+\mathrm{cj} .}
$$

mit

$$
f(z, \zeta)=S(z, \bar{z} ; \zeta) \log \frac{R(\zeta-z)}{\bar{z} \zeta-R^{2}}+\varphi(z, \zeta)
$$

und

$$
\varphi(z, \zeta)=\frac{1}{(1+\varepsilon z \bar{z})^{n}} \sum_{j=1}^{\infty} a_{j}(z, \bar{z}) \zeta^{j} .
$$

Für die Richtungsableitung auf $\dot{K}$ gilt sodann

$$
g_{v}=\frac{1}{R}\left\{z g_{z}+\bar{z} g_{\bar{z}}\right\}=-\frac{1}{4 \pi R}\left\{E\left(z f_{\tilde{z}}(z, \zeta)+\bar{z} f_{\bar{z}}(z, \zeta)+c_{j}\right\} .\right.
$$

Unter Verwendung der gemäss (49) konstruierten Greenschen Funktion und unter Berücksichtigung von Satz 2 erhält man eine verallgemeinerte Poissonsche Formel:

Satz 3. Für die reellwertige Funktion $w(\zeta, \bar{\zeta})$ gelte:

1. $w \in C^{1}$ in $\bar{K}$,

2. $w \in C^{2}$ in $K$,

3. $w$ sei Lösung von (1) in $K$.

Der Radius $R$ des Kreises $K$ genüge im Falle $\varepsilon=+1$ der Bedingung

$$
\sum_{k=0}^{j} A_{k}\left(\frac{R^{2}}{1+R^{2}}\right)^{n-k} \frac{1}{(j-k) !} \neq 0 \quad \text { für } \quad j=1, \ldots, n .
$$


Zunächst gilt

$$
-4 \pi g=2(E S)(z) \log \frac{R|z-\zeta|}{\mid \bar{\zeta} z-\overline{R^{2} \mid}}+T_{2}+\overline{T_{2}}+\{(E \varphi)(z)+\mathrm{cj} .\}
$$

mit

$T_{2}=$

$\sum_{k=1}^{n} A_{k}\left(\frac{\bar{z}}{1+\varepsilon z \bar{z}}\right)^{n-k} \sum_{\mu=1}^{n}\left(\begin{array}{l}k \\ \mu\end{array}\right) \frac{\partial^{k-\mu} S}{\partial z^{k-\mu}}(-1)^{\mu-1}(\mu-1) !\left\{\frac{1}{(z-\zeta)^{\mu}}-\frac{\bar{\zeta}^{\mu}}{\left(\bar{\zeta} z-R^{2}\right)^{\mu}}\right\}$.

Für alle $z \in \dot{K}$ gilt

$$
\log \frac{R^{2}(z-\zeta)(\bar{z}-\bar{\zeta})}{\left(\bar{\zeta} z-R^{2}\right)\left(\zeta \bar{z}-R^{2}\right)} \equiv 0
$$

wir haben also $\varphi(z)$ so zu bestimmen, dass

$$
\left\{T_{2}+(E \varphi)(z)\right\}_{|z|=R} \equiv 0 .
$$

Wir betrachten nun die in $|z| \leqq R$ definierte Funktion

$$
T_{3}=T_{3,1}-T_{3,2}
$$

mit

$$
\begin{aligned}
& T_{3,1}=\sum_{k=1}^{n} B_{k} z^{k} \sum_{\mu=1}^{k}\left(\begin{array}{l}
k \\
\mu
\end{array}\right) \frac{(-1)^{\mu-1}(\mu-1) !}{(z-\zeta)^{\mu}} \frac{\partial^{k-\mu} S}{\partial z^{k-\mu}}, \\
& T_{3,2}=\sum_{k=1}^{n} B_{k} z^{k} \sum_{\mu=1}^{k}\left(\begin{array}{l}
k \\
\mu
\end{array}\right) \frac{(-1)^{\mu-1}(\mu-1) ! \bar{\zeta}^{\mu}}{\left(\bar{\zeta} z-R^{2}\right)^{\mu}} \frac{\partial^{k-\mu} S}{\partial z^{k-\mu}}
\end{aligned}
$$

und

$$
B_{k}=A_{k}\left(\frac{R^{2}}{1+\varepsilon R^{2}}\right)^{n-k}
$$

Wegen

$$
\frac{\partial^{k-\mu} S}{\partial z^{k-\mu}}=\sum_{\varrho=0}^{n}\left(\begin{array}{l}
n \\
\varrho
\end{array}\right)\left(\frac{\varepsilon \bar{\zeta}}{1+\varepsilon \zeta \bar{\zeta}}\right)^{\varrho} \frac{(n+\varrho) !}{(n+\varrho+\mu-k) !}(z-\zeta)^{n+\varrho+\mu-k}
$$

folgt sodann

$$
T_{3,1}=\frac{1}{(1+\varepsilon \zeta \bar{\zeta})^{n}} \sum_{\eta=1}^{2 n} p_{\eta}\left(R^{2}, \zeta, \bar{\zeta}\right) z^{\eta}
$$


wobei die Koeffizienten $p_{\eta}$ Polynome in $\zeta, \bar{\zeta}$ darstellen. Wir entwickeln nun $T_{3,2}$ in eine Potenzreihe an der Stelle $z=0$. Dann folgt unter Berücksichtigung von

$$
\frac{1}{\left(\bar{\zeta} z-R^{2}\right)^{\mu}}=\frac{(-1)^{\mu}}{R^{2 \mu}} \sum_{j=0}^{\infty}\left(\begin{array}{c}
j+\mu-1 \\
\mu-1
\end{array}\right)\left(\frac{\bar{\zeta} z}{R^{2}}\right)^{j} \quad \text { für } \quad\left|\frac{\bar{\zeta} z}{R^{2}}\right|<1
$$

nach geeigneter Umformung und Zusammenfassung

$$
T_{3,2}=\frac{1}{(1+\varepsilon \zeta \bar{\zeta})^{n}}\left\{\sum_{\eta=1}^{3 n-1} q_{\eta}\left(R^{2}, \zeta, \bar{\zeta}\right) z^{\eta}+\sum_{\eta=3 n}^{\infty}\left(\frac{\bar{\zeta}}{R^{2}}\right)^{\eta-2 n} r_{\eta} z^{\eta}\right\}
$$

mit

$$
\begin{aligned}
& q_{\eta}=-\sum_{\substack{\tau=0 \\
\tau \leqq 2 n}}^{\eta-1}\left(\frac{\bar{\zeta}}{R^{2}}\right)^{\eta-\tau} C_{\tau} \sum_{\substack{k=1 \\
k \leqq n}}^{\eta} B_{k} \sum_{\substack{\mu=k-\tau \\
1 \leqq \mu \leqq-}}^{\eta-\tau} \frac{(\mu-1) !}{(\tau+\mu-k) !}\left(\begin{array}{c}
k \\
\mu
\end{array}\right)\left(\begin{array}{c}
\eta-\tau-1 \\
\mu-1
\end{array}\right), \\
& r_{\eta}=-\sum_{\tau=0}^{2 n}\left(\frac{\bar{\zeta}}{R^{2}}\right)^{2 n-\tau} C_{\tau} \sum_{k=1}^{n} B_{k} \sum_{\substack{k=k \\
k=\tau \\
\mu \geqq 1}}^{k} \frac{(\mu-1) !}{(\tau+\mu-k) !}\left(\begin{array}{c}
k \\
\mu
\end{array}\right)\left(\begin{array}{c}
\eta-\tau-1 \\
\mu-1
\end{array}\right)
\end{aligned}
$$

und

$$
C_{\tau}=\tau ! \sum_{\substack{i=\tau-k \\
0 \leqq \lambda \leqq n}}^{\tau}\left(\begin{array}{l}
n \\
\lambda
\end{array}\right)\left(\begin{array}{c}
n \\
\tau-\lambda
\end{array}\right)(-1)^{n-\lambda} \varepsilon^{\tau-\lambda} \zeta^{n-\lambda} \bar{\zeta}^{t-\lambda}
$$

Damit gilt

$$
\left|r_{n}\right|=O\left(\eta^{n-1}\right) \quad \text { für } \quad \eta \rightarrow \infty,
$$

wenn $\operatorname{man}$ in $r_{\eta}$ den Faktor

$$
\left(\begin{array}{c}
\eta-\tau-1 \\
\mu-1
\end{array}\right) \quad \text { und } \quad 1 \leqq \mu \leqq n
$$

berücksichtigt. Man erhält sodann unter Verwendung von (32) und (33) für $T_{3}$ eine in $|z|<R^{2} /|\zeta|$ konvergente und für $\zeta=0$ abbrechende Reihe

$$
T_{3}=\frac{1}{(1+\varepsilon \zeta \bar{\zeta})^{n}} \sum_{j=1}^{\infty} b_{j} z^{j}
$$

Hilfssatz 1. Es sei

$$
d_{j}=\sum_{\substack{k=0 \\ k \leqq n}}^{j} B_{k} \frac{j !}{(j-k) !}, \quad B_{k}=A_{k}\left(\frac{R^{2}}{1+\varepsilon R^{2}}\right)^{n-k},
$$

mit 


$$
d_{j} \neq 0 \quad \text { für } \quad 1 \leqq j \leqq n \text { und } \varepsilon=1 .
$$

Dann gibt es eine in $|z| \leqq R$ definierte Lösung $\varphi(z)$ der inhomogenen Eulerschen Differentialgleichung

$$
\sum_{k=0}^{n} B_{k} z^{k} \varphi^{(k)}(z)+T_{3}(z)=0
$$

mit

$$
\begin{gathered}
T_{3}(z)=\sum_{k=1}^{n} B_{k} z^{k} \sum_{\mu=1}^{k}\left(\begin{array}{l}
k \\
\mu
\end{array}\right)(-1)^{\mu-1}(\mu-1) ! \frac{\partial^{k-\mu} S}{\partial z^{k-\mu}}\left(\frac{1}{(z-\zeta)^{u}}-\frac{\overline{\zeta^{\prime \prime}}}{\left(\bar{\zeta} z-R^{2}\right)^{u}}\right), \\
S=\frac{(z-\zeta)^{n}(1+\varepsilon \bar{\zeta} z)^{n}}{(1+\varepsilon \zeta \bar{\zeta})^{n}}, \quad|\zeta|<R,
\end{gathered}
$$

und es gilt mit (28) und (29) $g \mid \dot{K} \equiv 0$.

Beweis. Wir setzen formal

$$
\varphi(z)=\frac{1}{(1+\varepsilon \zeta \bar{\zeta})^{n}} \sum_{j=1}^{\infty} a_{j} z^{j} .
$$

Dann folgt durch Koeffizientenvergleich, wie eine elementare Rechnung zeigt

$$
a_{j}=-\frac{b_{j}}{d_{j}} .
$$

$\mathrm{Da}$ in (39) für $\varepsilon=-1$ alle Summanden positiv sind, gilt in diesem Falle allgemein

$$
d_{j} \neq 0, \quad j \in N .
$$

Im Falle $\varepsilon=+1$ ist dies für $j=1, \ldots, n$ durch (40) gesichert. Durch diese Bedingung werden genau die in Satz 1 genannten Fälle ausgeschlossen, in denen das Dirichlet-Problem für den Kreis nicht eindeutig lösbar ist. Damit ist zugleich

$$
d_{j} \neq 0 \text { für } \varepsilon=+1 \text { und } n<j<2 n
$$

gesichert, wie man durch Umformung nachweist oder unmittelbar aus Satz $\mathrm{V}$ folgern kann. Im Falle $j \geqq 2 n$ betrachten wir die durch

$$
h(z)=z^{j}, \quad j \geqq 2 n,
$$

erzeugte Lösung

$$
w=(E h)(z)=z^{j-n} \sum_{k=0}^{n} \frac{j ! A_{k}}{(j-k) !}\left(\frac{z \bar{z}}{1+z \bar{z}}\right)^{n-k} .
$$


Dane gilt:

$$
w(\zeta, \bar{\zeta})=-\int_{|z|=R} w(z, \bar{z}) g_{v}(\zeta, \bar{\zeta} ; z, \bar{z}) d s
$$

mit $g_{.}$gemäss (49) bis (52).

7. Der Poissonsche Satz. Wir haben bei Satz 3 vorausgesetzt, dass eine bekannte in $K$ definierte reellwertige Lösung von (1) vorliegt, die auf $\dot{K}$ noch differenzierbar ist. Eine solche Funktion lässt sich sodann gemäss (53) vermöge ihrer Randwerte auf $\dot{K}$ darstellen. Wir gehen nun umgekeht davon aus, dass auf $\dot{K}$ eine stetige reellwertige Funktion $u(z, z)$ gegeben ist, und zeigen, dass dazu eine Funktion $w$ existiert, die in $\dot{K}$ Lösung von (1) ist.

Satz 4. Die reellwertige Funktion $u(z, \bar{z})$ sei stetig auf $|z|=R$. Dabei genüge $R$ im Falle $\varepsilon=+1$ der Bedingung

$$
\sum_{k=1}^{j} A_{k}\left(\frac{R^{2}}{1+R^{2}}\right)^{n-k} \frac{1}{(j-k) !} \neq 0 \text { für } j=1, \ldots, n .
$$

a) Dann stelli

$$
w(\zeta, \bar{\zeta})= \begin{cases}-\int_{\mid z^{\prime}=R} u(z, \bar{z}) g_{\nu}(\zeta, \bar{\zeta} ; z, \bar{z}) d s & \text { für }|\zeta|<R \\ u(\zeta, \bar{\zeta}) & \text { für }|\zeta|=R\end{cases}
$$

eine in $-\mid \leqq R$ stetige Funktion dar, die für $|\zeta|<R$ der Differentialgleichung (1) genügt.

b) Die durch (54) gegebene Funktion stellt die einaige in $|\zeta|<R$ definierte Lösung von (1) dar, die auf $|\zeta|=R$ die vorgegebenen Randwerte besitzt.

c) $w(\zeta, \bar{\zeta})$ stellt eine stetige Funktion $\left.{ }^{5}\right)$ der Randwerte dar.

Zum Beweis dieses verallgemeinerten Poissonschen Satzes zeigen wir zunächst, dass die durch (54) gegebene Funktion eine Lösung von (1) in $|\zeta|<R$ ist. Für die Greensche Funktion gilt gemäss (49)

`) Bezeichnet man den Raum der auf $\{z \mid=R$ stetigen reellwertigen Funktionen $u(z, \bar{z})$ mit $U$, den Raum der in $|\zeta|<R$ definierten reellwertigen und in $|\zeta| \leqq R$ stetig ergänzbaren Lösungen $w(\zeta, \bar{\zeta})$ von (1) mit $W$ und versieht man $U$ bzw. $W$ mit den Metriken

$$
d\left(u_{1}, u_{2}\right)=\max \left|u_{1}-u_{2}\right| \quad \text { bzw. } \quad e\left(w_{1}, w_{2}\right)=\max \left|w_{1}-w_{2}\right|,
$$

so besagt die unter c) formulierte Aussage, dass mit (54) eine stetige Abbildung von $U$ in $W$ gegeben ist. 
(59)

$$
\dot{K}_{1}=\left\{z|z \in \dot{K},| z-z_{0} \mid \leqq \varrho\right\}
$$

und

$$
\dot{K}=\dot{K}_{1}+\dot{K}_{2} .
$$

Dann folgt mit (54) und $u_{0}=u\left(z_{0}, \bar{z}_{0}\right)$

$$
w-u_{0}=-\int_{|z|=R}\left(u-u_{0}\right) g_{\nu} d s=I_{1}+I_{2}
$$

mit

$$
I_{1}=-\int_{\dot{K}_{1}}\left(u-u_{0}\right) g_{v} d s, \quad I_{2}=-\int_{\dot{K}_{2}}\left(u-u_{0}\right) g_{v} d s
$$

und es gilt

$$
\left|I_{1}\right| \leqq\left|\frac{\omega}{C}\right| \max _{z \in \dot{K}_{1}}\left(\left|u-u_{0}\right|\right) .
$$

Da $\omega$ eine in $|\zeta| \leqq R$ stetige Funktion darstellt, gibt es eine Konstante $c>0$, so dass

$$
|\omega| \leqq c \quad \text { für } \quad|\zeta| \leqq R .
$$

Damit folgt

$$
\left|I_{1}\right| \leqq \frac{c}{|C|} \max _{z \in \dot{K}_{1}}\left(\left|u-u_{0}\right|\right) .
$$

Für die Greensche Funktion gilt

$$
\begin{aligned}
-4 \pi g & =\underset{z}{E}(f(\zeta, z))+\mathrm{cj} . \\
& \left.=2(E S)(z) \log \frac{R|z-\zeta|}{\mid \bar{\zeta} z-\overline{R^{2} \mid}}+T_{2}+\overline{T_{2}}+\underset{z}{\{}(\varphi(\zeta, z))+\mathrm{cj} .\right\} .
\end{aligned}
$$

Mit

$$
L=\log \frac{R(z-\zeta)}{\zeta z-R^{2}}
$$

folgt sodann

$$
L_{z}=\frac{\zeta \bar{\zeta}-R^{2}}{(z-\zeta)\left(\bar{\zeta} z-R^{2}\right)}
$$

sowie

$$
T_{2}=\sum_{k=1}^{n} A_{k}\left(\frac{\bar{z}}{1+\varepsilon z \bar{z}}\right)^{n-k} \sum_{\mu=1}^{k}\left(\begin{array}{l}
k \\
\mu
\end{array}\right) \frac{\partial^{k-\mu} S}{\partial z^{k-\mu}} \frac{\partial^{\mu} L}{\partial z^{\mu}}=\left(\zeta \bar{\zeta}-R^{2}\right) T_{5},
$$


A. I. 437

wobei $T_{5}$ eine in $|z| \leqq R,|\zeta| \leqq R$ stetige Funktion darstellt. Entsprechend folgt für die in (31) definierte Grösse $T_{3}$ :

$$
T_{3}=\left(\zeta \bar{\zeta}-R^{2}\right) T_{6}
$$

mit einer in $|z| \leqq R,|\zeta| \leqq R$ stetigen Funktion $T_{6}$. Damit lässt sich auch bei der Lösung $\varphi(z)$ von (41) ein Faktor $\left(\zeta \bar{\zeta}-R^{2}\right)$ abspalten und es gilt

$$
-4 \pi g=2(E S)(z) \log \frac{R|z-\zeta|}{\left|\bar{\zeta} z-R^{2}\right|}+\left(\zeta \bar{\zeta}-R^{2}\right) \Psi(\zeta, \bar{\zeta} ; z, \bar{z}),
$$

wobei $\Psi$ eine für alle $|\zeta| \leqq R$ stetige und für $|z| \leqq R$ nach $z$ und $\bar{z}$ differenzierbare Funktion darstellt. Durch eine elementare Rechnung folgt sodann für $|z|=R$ :

$$
g_{\nu} \mid \dot{K}=\frac{R^{2}-\zeta \bar{\zeta}}{4 \pi R}\left\{\frac{(E S)(z)}{|z-\zeta|^{2}}+z \Psi_{z}+\bar{z} \Psi_{\bar{z}}\right\}_{\mid z^{\prime}=R} .
$$

Wir könneı nun ohne Beschränkung der Allgemeinheit annehmen, dass bereits

$$
\left|\zeta-z_{0}\right|<\frac{\varrho}{2}
$$

dann gilt:

$$
|z-\zeta| \geqq\left|z-z_{0}\right|-\left|z_{0}-\zeta\right| \geqq \varrho-\frac{\varrho}{2}=\frac{\varrho}{2} .
$$

Ausserdem gilt für $z \in \dot{K}_{2}$

$$
\begin{gathered}
g_{1} \leqq \frac{(R-|\zeta|)(R+|\zeta|)}{4 \pi R|z-\zeta|^{2}}|(E S)(z)+| z-\left.\zeta\right|^{2}\left(z \Psi_{z}+\bar{z} \Psi_{\bar{z}}\right) \mid \\
\leqq \frac{2 M_{1}(R-|\zeta|)}{\pi \varrho^{2}}
\end{gathered}
$$

mit

$$
M_{1}=\max _{\substack{|z| \leq R \\|j| \leqq R}}\left\{|(E S)(z)+| z-\left.\zeta\right|^{2}\left(z \Psi_{z}+\bar{z} \Psi_{z}\right)\right\}
$$

Setzt man ausserdem

$$
M_{2}=\max _{z \in \dot{K}}(|u(z, \bar{z})|),
$$

so folgt

$$
\left|u-u_{0}\right| \leqq|u|+\left|u_{0}\right| \leqq 2 M_{2}
$$


und damit

$$
\left|I_{2}\right| \leqq \frac{8 M_{1} M_{2} R}{\varrho^{2}}(R-|\zeta|) .
$$

Zusammenfassend gilt also

$$
w-u_{0} \mid \leqq \frac{c}{|C|} \max _{z \in \dot{K}_{1}}\left(\left|u-u_{0}\right|\right)+\frac{8 M_{1} M_{2} R}{Q^{2}}(R-|\zeta|) .
$$

Wegen der Stetigkeit der Funktion $u$ können wir bei vorgegebenem $\varepsilon^{*}>0$ ein $\varrho$ derart wählen, dass

$$
\frac{c}{|C|} \max _{z \in \dot{K_{1}}}\left(\left|u-u_{0}\right|\right)<\frac{\varepsilon^{*}}{2} .
$$

Unter Verwendung eines derart festgelegten Wertes für $\varrho$ können wir nun ein $\delta\left(\varepsilon^{*}\right)>0$ angeben, so dass

$$
\frac{8 M_{1} M_{2} R}{\varrho^{2}}(R-\zeta)<\frac{\varepsilon^{*}}{2} \quad \text { für }\left|\zeta-z_{0}\right|<\delta\left(\varepsilon^{*}\right),
$$

da

$$
R-|\zeta|=\left|z_{0}\right|-|\zeta| \leqq\left|\zeta-z_{0}\right| \text {. }
$$

Damit folgt abschliessend

$$
\left|w-u_{0}\right|<\varepsilon^{*} \quad \text { für } \quad\left|\zeta-z_{0}\right|<\delta\left(\varepsilon^{*}\right) .
$$

Die durch (54) definierte Funktion ist also in $\zeta \leqq R$ stetig und nimmt die Randwerte $u(z, \bar{z})$ auf $|\zeta|=R$ an.

Die Eindeutigkeit der Lösung folgt nun sofort mit Satz 1, da die im Falle $\varepsilon=+1$ geforderten Bedingungen für den Radius $R$ hier nach Voraussetzung erfüllt sind.

Es seien nun $w_{1}$ bzw. $w_{2}$ Lösungen des vorliegenden Randwertproblems mit den Randwerten $u_{1}(z, \bar{z})$ bzw. $u_{2}(z, \bar{z})$. Dann gilt unter Verwendung von (54)

$$
\left|w_{1}-w_{2}\right| \leqq \max _{|z|=R}\left(\left|u_{1}-u_{2}\right|\right) \int_{z=K} g_{v} d s:
$$

Berücksichtigt man ausserdem (58) und (61), so folgt

$$
\left|w_{1}-w_{2}\right| \leqq \frac{c}{|C|} \max _{|z|=R}\left(\left|u_{1}-u_{2}\right|\right),
$$

womit auch die Aussage c) des Satzes 4 bewiesen ist. 
8. Die Schwarzsche Formel. In der klassischen Funktionentheorie liefert die bekannte Schwarzsche Formel (vgl. etwa [7])

$$
f(\zeta)=\frac{1}{2 \pi} \int_{0}^{2 \pi} u\left(R e^{i_{\varphi}}\right) \frac{z+\zeta}{z-\zeta} d \varphi+i v(0)
$$

die Darstellung einer in $|\zeta|<R$ holomorphen Funktion $f(\zeta)=u(\zeta)+$ $i v(\zeta)$ mit Hilfe der Randwerte $u\left(R e^{i \varphi}\right)$ ihres Realteils, wenn der Wert des Imaginärteils $v$ an der Stelle $\zeta=0$ vorgegeben wird.

Im Falle der Lösungen der Differentialgleichung (1) lässt sich eine ähnliche Aussage für die den holomorphen Funktionen entsprechenden Lösungen (vgl. [1])

$$
w=(E h)(z)
$$

gewinnen, da man den Realteil einer solchen Funktion als Poisson-Integral darstellen kann.

Wir nehmen zunächst an, es liege eine in einem einfach zusammenhängenden Gebiet $G$ definierte Lösung

$$
w^{*}=\left(E h^{*}\right)(z)
$$

von (1) vor. Jede andere in $G$ definierte Lösung

$$
w=(E h)(z)
$$

mit dem gleichen Realteil erhält man sodann nach Satz IIIa unter Verwendung der Erzeugenden

$$
h(z)=h^{*}(z)+P(z),
$$

wobei $P(z)$ ein Polynom vom Grade $2 n$ in $z$ mit

$$
P(z)+(-\varepsilon)^{n} z^{2 n} P\left(\frac{-\varepsilon}{\bar{z}}\right)=0
$$

darstellt. Setzt man

$$
P(z)=\sum_{\mu=0}^{2 n} c_{\mu} z^{\mu}, \quad c_{\mu} \in C,
$$

so ist die Bedingung (65) gleichbedeutend mit

$$
c_{\mu}+(-\varepsilon)^{n+\mu} \overline{c_{2 n-\mu}}=0
$$

und man erhält

$$
(E P)(z)=\sum_{\mu=0}^{n-1}\left(c_{\mu} \bar{z}^{n-\mu}-\overline{c_{\mu}} z^{n-\mu}\right) B_{\mu}+c_{n} B_{n}
$$

mit 


$$
B_{\mu}=\sum_{\tau=0}^{\mu} \frac{(z \bar{z})^{\mu-\tau}(-\varepsilon)^{n+\tau} n !}{(1+\varepsilon z \bar{z})^{n}}\left(\begin{array}{c}
\mu \\
\tau
\end{array}\right)\left(\begin{array}{c}
2 n-\mu \\
n-\tau
\end{array}\right), \quad \mu=0,1, \ldots, n .
$$

Eine bestimmte Lösung der durch (64) gegebenen Schar kann dadurch festgelegt werden, dass man ihren Imaginärteil in gewissen in $G$ liegenden Punkten vorschreibt. Setzt man nämlich

$$
\begin{aligned}
& c_{\prime \prime}=\alpha_{\mu}+i \beta_{\mu}, \quad \mu=0,1, \ldots, n-1, \\
& c_{n}=i \beta_{n} \quad \text { mit } \quad \alpha_{\mu}, \beta_{\mu}, \beta_{n} \in R
\end{aligned}
$$

und schreibt den Imaginärteil von $w$ in $z=z_{1}$ vor, so erhält man unter Verwendung von (64) und (66) eine Bestimmungsgleichung (im Reellen) für die $2 n+1$ Unbekannten

$$
\alpha_{0}, \ldots, \alpha_{n-1}, \beta_{0}, \ldots, \beta_{n-1}, \beta_{n} .
$$

Damit wird die Funktion $w$ eindeutig festgelegt, wenn man die Imaginärteile in $2 n+1$ geeignet gewählten Punkten $z_{1}, \ldots, z_{2 n+1}$ vorschreibt. Aufgrund von (66) erkennt man sofort, dass die Grössen $c_{\mu}$ nicht eindeutig bestimmt werden, wenn man die Imaginärteile in $2 n+1$ Punkten vorschreibt, die sämtlich auf der reellen Achse liegen. In diesem Fall können die Grössen $\alpha_{u}, \mu=0,1, \ldots, n-1$, noch beliebig gewählt werden. Da die Differentialgleichung invariant gegenüber Kugeldrehungen $(\varepsilon=+1)$ bzw. Automorphismen des Einheitskreises $(\varepsilon=-1)$ ist, erhält man die notwendige Bedingung, dass die Punkte $z_{1}, \ldots, z_{2 n+1}$, in denen die Imaginärteile vorgeschrieben werden, nicht sämtlich auf einem Grosskreis der Riemannschen Zahlenkugel $(\varepsilon=+1)$ oder einem Orthogonalkreis des Einheitskreises $(\varepsilon=-1)$ liegen dürfen. Im Falle $n=1$ ist diese Bedingung (für $\varepsilon= \pm 1$ ) auch hinreichend.

Im Falle $\varepsilon=+1$ werden die Koeffizienten $c_{\text {" }}$ auch dann nicht mehr eindeutig bestimmt, wenn man die Punkte $z_{1}, \ldots, z_{2 n+1}$ auf einem Kreis $z=r$ wählt, für den eine oder mehrere der Grössen $B_{u}$ verschwinden. Wie man leicht nachrechnet, handelt es sich hier genau um die Kreise, für die das oben behandelte Randwertproblem im Falle $\varepsilon=+1$ nicht mehr eindeutig lösbar ist. Damit gilt der folgende

Hilfssatz 2. a) Ist eine im einfach zusammenhängenden Gebiet $G$ definierte Lösung

$$
w^{*}=\left(E h^{*}\right)(z)
$$

von (1) gegeben, so erhält man alle in $G$ definierten Lösungen dieser Art mit gleichem Realteil gemäss

$$
w=(E h)(z)
$$




$$
h(z)=h^{*}(z)+P(z)
$$

und

$$
P(z)=\sum_{n=0}^{2 n} c_{\mu} z^{\mu}, \quad c_{\mu}+(-\varepsilon)^{n+\mu} \overline{c_{2 n-\mu}}=0 .
$$

b) Eine Lösung $w$ der Schar (67) wird eindeutig festgelegt, wenn man den Imaginärteil von $w$ in geeignet gewählten Punkten $z_{1}, \ldots, z_{2 n-1}$ vorschreibt.

c) Dabei gilt als notwendige Bedingung im Falle $\varepsilon=-1$, dass die Punkte $z_{1}, \ldots, z_{2 n+1}$ nicht sämtlich auf einem Orthogonalkreis liegen. Im Falle $\varepsilon=+1$ dürfen die genannten Punkte weder auf einem Grosskreis der Riemannschen Zahlenkugel noch auf einem Kreis $|z|=r$ mit

$$
\sum_{k=0}^{j} A_{k}\left(\frac{r^{2}}{1+r^{2}}\right)^{n-k} \frac{1}{(j-k) !} \neq 0 \quad \text { für } j=1, \ldots, n
$$

liegen.

Sei nun $u(z, \bar{z})$ eine reellwertige und auf $|z|=R$ stetige Funktion. Dabei gelte im Falle $\varepsilon=+1$

$$
\sum_{k=0}^{j} A_{k}\left(\frac{R^{2}}{1+R^{2}}\right)^{n-k} \frac{1}{(j-k) !} \neq 0 \quad \text { für } \quad j=1, \ldots, n .
$$

Dann erhält man durch

$$
w^{*}(\zeta, \bar{\zeta})=\frac{1}{2 \pi R} \int_{|z|=R} u(z, \bar{z}) \underset{\xi}{E}\left(z f_{z}(z, \zeta)+\bar{z} f_{\bar{z}}(z, \zeta)\right) d s
$$

mit $f(z, \zeta)$ gemäss $(50)$ eine in $|\zeta|<R$ definierte Lösung

$$
w^{*}(\zeta, \bar{\zeta})=\left(E h^{*}\right)(\zeta)
$$

von (1) mit dem Realteil

$u(\zeta, \bar{\zeta})=\operatorname{Re}\left\{w^{*}\right\}=\frac{1}{4 \pi R} \int_{|z|=R} u(z, \bar{z})\left(E\left(z f_{z}(z, \zeta)+\bar{z} f_{\bar{z}}(z, \zeta)\right)+\mathrm{cj}.\right) d s$.

Nach Satz IIIa erhält man jede andere in $|\zeta|<R$ definierte Lösung dieser Art mit gleichem Realteil durch

$$
w(\zeta, \bar{\zeta})=\frac{1}{2 \pi R} \int_{|z|=R} u(z, \bar{z}) \underset{\zeta}{E}\left(z f_{z}(z, \zeta)+\bar{z} f_{\bar{z}}(z, \zeta)\right) d s+(E P)(\zeta)
$$

Berücksichtigt man hier noch das im Hilfssatz 2 gewonnene Ergebnis, so erhält man den folgenden 
Satz 5. Sei $u(z, \bar{z})$ eine auf $|z|=R$ stetige reellwertige Funktion. Im Falle $\varepsilon=+1$ gelte

$$
\sum_{k=0}^{j} A_{k}\left(\frac{R^{2}}{1+R^{2}}\right)^{n-k} \frac{1}{(j-k) !} \neq 0 \quad \text { für } j=1, \ldots, n .
$$

a) Dann lässt sich jede in $|\zeta|<R$ definierte Lösung

$$
w=(E h)(\zeta)
$$

von (1), deren Realteil auf $|\zeta|=R$ die Randwerte $u(\zeta, \bar{\zeta})$ annimmt, in der Form

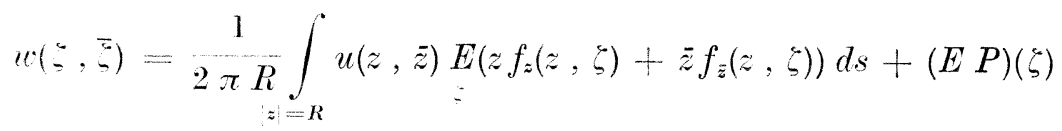

mit $P(\zeta)$ gemäss (68) und $f(z, \zeta)$ gemäss (50) darstellen.

b) Eine Lösung der Form (69) mit dieser Eigenschaft wird eindeutig festgelegt, wenn man den Imaginärteil in $2 n+1$ geeignet gewählten Punkten vorschreibt.

Technische Hochschule in Graz

Österreich 


\section{Literatur}

[1] BAUer, K. W.: Über eine der Differentialgleichung $(1 \pm z \bar{z})^{2} w_{z \bar{z}} \pm n(n+1) w$ $=0$ zugeordnete Funktionentheorie. - Bonner math. Schriften 23, 1965.

[2] -"- Über die Lösungen der elliptischen Differentialgleichung $(1 \pm z \bar{z})^{2} w_{z \bar{z}}$ $+\lambda w=0$. Teil I. - J. reine angew. Math. 221, 1966, S. 48-84.

[3] - - Über die Lösungen der elliptischen Differentialgleichung $(1 \pm z \bar{z})^{2} w_{z \bar{z}}$ $+\lambda w=0$. Teil II. - J. reine angew. Math. 221, 1966, S. 176-196.

[4] Bauer, K. W., und E. Peschl: Ein allgemeiner Darstellungssatz für die Lösungen der Differentialgleichung $(1+\varepsilon z \bar{z})^{2} w_{z \bar{z}}+\varepsilon n(n+1) w=0$ in der Nähe isolierter Singularitäten. - Bayer. Akad. Wiss., math.-naturw. Kl., S.-ber. 1965,1966 , S. $113-146$.

[5] ГАнин, М. П. [M. Р. Ganin]: Задача Дирихле для уравнения $\Delta U+\frac{4 n(n+1)}{\left(1+x^{2}+y^{2}\right)^{2}} U=0$. - Успехи мат. наук $12: 5(77), 1957$, S. $205-209$.

[6] Hellwig, G.: Partielle Differentialgleichungen. Eine Einführung. - Mathematische Leitfäden, B. G. Teubner Verlagsgesellschaft, Stuttgart, 1960.

[7] Nevandinna, R., und V. Paatero: Einführung in die Funktionentheorie. - Lehrbücher und Monographien aus dem Gebiete der exakten Wissenschaften, Mathematische Reihe 30, Birkhäuser Verlag, Basel / Stuttgart, 1965.

[8] Roelcke, W.: Über die Wellengleichung bei Grenzkreisgruppen erster Art. S.-ber. Heidelberger Akad. Wiss., math.-naturw. Kl. 1953/1955, 1956, S. $159-267$.

[9] Vekua, I. N.: New methods for solving elliptic equations. - North-Holland Series in Applied Mathematics and Mechanics 1, North-Holland Publishing Company / John Wiley \& Sons, Inc. (Interscience Publishers Division), Amsterdam / New York, 1967. 